\title{
Do women benefit more than men from off-pump coronary artery bypass grafting?
}

\author{
J. F. ter Woorst · A. H. T. Hoff · M. C. Haanschoten - S. Houterman - A. H. M. van Straten · M. A. Soliman-Hamad
}

Published online: 20 September 2019

(C) The Author(s) 2019

\begin{abstract}
Objectives Outcomes after coronary artery bypass grafting $(\mathrm{CABG})$ are worse in women than in men. This study aims to investigate whether off-pump coronary artery bypass (OPCAB) surgery improves the outcomes in women by comparing different outcome measures in both genders.

Methods Patients who underwent isolated CABG, either on-pump (ONCAB) or OPCAB, between January 1998 and June 2017 were included. Primary endpoints were 30-day and 120-day mortality. Logistic regression models were constructed to evaluate the effect of the CABG technique on important outcomes such as mortality and the need for blood transfusion.

Results The data of 17,052 patients were analysed, 3,684 of whom were women (414 OPCAB) and 13,368 men $(1,483$ OPCAB). The mean number of grafts was lower in the OPCAB group of both genders $(p<0.001)$. Postoperatively, both men and women undergoing OPCAB surgery received fewer red blood cell transfusions $(p<0.001)$ and had higher postoperative haemoglobin levels $(p<0.001)$ than those undergoing ONCAB. Early mortality occurred less frequently after OPCAB surgery in both genders, although the difference was not significant. However, 120-day mortality was significantly lower after OPCAB surgery in
\end{abstract}

\footnotetext{
J. F. ter Woorst · A. H. T. Hoff · A. H. M. van Straten •

M. A. Soliman-Hamad $(\bowtie)$

Department of Cardiothoracic Surgery, Catharina Hospital, Eindhoven, The Netherlands

mohammed.soliman@catharinaziekenhuis.nl

M. C. Haanschoten

Department of Anaesthesiology, and Intensive Care Unit, Catharina Hospital, Eindhoven, The Netherlands

S. Houterman

Department of Education and Research, Catharina Hospital, Eindhoven, The Netherlands
}

women, even after correction for preoperative risk factors [odds ratio $(\mathrm{OR})=0.356,95 \%$ confidence interval (CI) $0.144-0.882, p=0.026$ ]. The difference in 120day mortality was not significant in men $(\mathrm{OR}=0.787$, 95\% CI 0.498-1.246, $p=0.307$ ).

Conclusions Women undergoing CABG benefit more from OPCAB surgery than from ONCAB surgery in terms of 120-day mortality. This difference was not found in men in our patient population.

Keywords Coronary artery bypass grafting • Offpump coronary artery bypass · On-pump coronary artery bypass - Statistics · Regression analysis . Gender

\section{Introduction}

Female patients have higher operative morbidity and mortality than males after coronary artery bypass grafting (CABG) [1-4]. It has been claimed that these variations in outcomes could be attributed to different revascularisation strategies [5]. Previous studies have also shown that women who underwent CABG were

\section{What's new?}

- In female patients undergoing coronary artery bypass grafting (CABG), the adjusted 120-day mortality after off-pump CABG is significantly lower than that after on-pump CABG. This finding was not demonstrated in the male population undergoing CABG.

- In both male and female populations undergoing CABG, a lower rate of postoperative blood transfusions and a higher postoperative haemoglobin level are demonstrated after the off-pump technique compared to the on-pump technique. 
older and had more co-morbidities, such as diabetes, hyperlipidaemia, hypertension and congestive heart failure, than men [1, 4, 6-9]. Moreover, female gender is scored as a risk factor for adverse outcomes after CABG in frequently used scoring systems, such as the European System for Cardiac Operative Risk Evaluation (EuroSCORE) [10] and the Society of Thoracic Surgeons (STS) [11] score. Off-pump coronary artery bypass grafting (OPCAB) has been shown to improve the outcome particularly in high-risk patients [12]. Some previous studies have shown a beneficial effect of OPCAB surgery for women $[2,3,6,13,14]$. Although much research has been conducted concerning the outcomes after ONCAB and OPCAB surgery in women, results are conflicting and no recent data are available. Also, previous research has mainly focused on early and in-hospital mortality, which might not be an accurate outcome measurement for cardiac surgery $[15,16]$. The aim of the present study was to determine if women benefit more from OPCAB than from ONCAB surgery by comparing the outcomes of these techniques in both genders.

\section{Methods}

This study included all patients who underwent isolated CABG, either ONCAB or OPCAB, between January 1998 and June 2017 at our institution (Catharina Hospital Eindhoven, The Netherlands). In order to minimise the heterogeneity of the population, patients were excluded if they had single-vessel disease, if they had undergone prior cardiac surgery, or if they had had an on-pump beating heart operation without aortic cross clamping. Clinical data, including patient demographics, complications and mortality, were extracted from a computerised database of the Department of Cardiothoracic Surgery. Preoperative variables included age, underweight [body mass index (BMI) $<20 \mathrm{~kg} / \mathrm{m}^{2}$ ], obesity (BMI $\geq 30 \mathrm{~kg} / \mathrm{m}^{2}$ ), hypertension, chronic obstructive pulmonary disease (COPD), peripheral vascular disease (PVD), diabetes mellitus, left ventricular ejection fraction (LVEF) $<35 \%$, haemoglobin level, estimated glomerular filtration rate (eGFR, calculated using the Modification of Diet in Renal Disease formula [17]) and triple-vessel disease. The ethics committee of our institution approved our study protocol and waived the need for informed consent.

The primary endpoints were early mortality (within 30 days postoperatively) and 120-day mortality, data obtained from the municipal administration (Gemeentelijke Basisadministratie). Secondary in-hospital endpoints included cerebrovascular accident (CVA), transient ischaemic attack (TIA)/reversible ischaemic neurological deficit (RIND), myocardial infarction, blood product transfusions ( $\geq 1$ packed cells) during hospital stay, re-exploration for bleeding and postoperative haemoglobin level (data obtained the day before discharge, 2-5 days after surgery).
The decision to use the off-pump technique was made by the surgeon and did not follow certain selection criteria. Our standard policy to stop preoperative anticoagulant therapy was applied to the whole patient population throughout the study period. In patients receiving preoperative dual anti-platelet therapy, acetylsalicylic acid (aspirin) was continued until the time of the operation, while P2Y12 inhibitors were stopped 5 days preoperatively.

In both ONCAB and OPCAB surgery, all patients received short-acting anaesthesia to facilitate rapid extubation. In ONCAB surgery, the extracorporeal circulation (ECC) was normothermic with a non-pulsatile flow; cardioplegic arrest was accomplished by the use of cold crystalloid cardioplegia (St. Thomas' solution) or warm blood cardioplegia, according to the surgeon's preference. In OPCAB surgery a gauze sling was temporarily fixed to the deepest point of the pericardium for maximum exposure of the heart or a vacuum-assisted apical suction device (Starfish, Medtronic, Eindhoven, The Netherlands) was used. A suction device (Octopus, Medtronic) to immobilise the target vessel and an intracoronary shunt (ClearView Shunt, Medtronic) were used. Since 2003, cell salvage has been used in all patients undergoing CABG, whether OPCAB or ONCAB surgery.

\section{Statistical analysis}

Patients were divided into groups based on gender and subsequently divided into subgroups based on the type of surgery (ONCAB or OPCAB). To compare categorical variables, the chi-square or Fisher's exact test was used, and figures are presented as numbers and percentages. Skewness and kurtosis were used to explore normality for continuous variables. Normally distributed variables were compared by the independent samples $t$-test and are presented as mean \pm standard deviation (SD). Non-normally distributed variables were compared by the MannWhitney test and are presented as median and interquartile range (IQR, $25 \%$ and $75 \%$ ). To identify the effect of OPCAB surgery on significant postoperative outcomes, separate regression analyses were performed. In these models, type of surgery was the independent variable with ONCAB as reference; the outcome was the dependent variable. For the categorical outcomes early mortality, 120-day mortality, red blood cell transfusion and re-exploration, logistic regression was performed. For the continuous outcome postoperative haemoglobin level, linear regression analysis was performed. When odds ratios in the univariate analysis were significant, a multivariable analysis was performed with correction for differences in preoperative variables. A $p$ value of less than 0.05 was considered significant. All statistical analyses were performed using IBM SPSS Statistics 24 (SPSS Inc., Chicago, IL, USA). 
Table 1 Preoperative characteristics of patients undergoing on-pump coronary artery bypass (ONCAB) and off-pump coronary artery bypass $(O P C A B)$ surgery

\begin{tabular}{|c|c|c|c|c|c|c|}
\hline \multirow[t]{2}{*}{ Variable } & \multicolumn{3}{|l|}{ Women $(n=3,684)$} & \multicolumn{3}{|l|}{$\operatorname{Men}(n=13,368)$} \\
\hline & $\begin{array}{l}\text { ONCAB } \\
(n=3,270)\end{array}$ & OPCAB $(n=414)$ & $p$-value & $\begin{array}{l}\text { ONCAB } \\
(n=11,885)\end{array}$ & OPCAB $(n=1,483)$ & $p$-value \\
\hline Age (years), mean $\pm S D$ & $68.8 \pm 8.8$ & $67.6 \pm 10.2$ & 0.021 & $64.7 \pm 9.4$ & $64.0 \pm 10.1$ & 0.011 \\
\hline Underweight $\left(\mathrm{BMI}<20 \mathrm{~kg} / \mathrm{m}^{2}\right)$ & $78(2.4 \%)$ & $5(1.2 \%)$ & 0.128 & $94(0.8 \%)$ & $17(1.1 \%)$ & 0.155 \\
\hline Obese $\left(\mathrm{BMI} \geq 30 \mathrm{~kg} / \mathrm{m}^{2}\right)$ & $886(27.1 \%)$ & $132(31.9 \%)$ & 0.040 & $2,349(19.8 \%)$ & $293(19.8 \%)$ & 0.995 \\
\hline Hypertension & $1,930(59.0 \%)$ & $259(62.6 \%)$ & 0.167 & $5,474(46.1 \%)$ & $732(49.4 \%)$ & 0.016 \\
\hline COPD & $398(12.2 \%)$ & $44(10.6 \%)$ & 0.363 & $1,253(10.5 \%)$ & $144(9.7 \%)$ & 0.323 \\
\hline PVD & $422(12.9 \%)$ & $60(14.5 \%)$ & 0.367 & $1,469(12.4 \%)$ & $200(13.5 \%)$ & 0.216 \\
\hline Prior CVA & $151(4.6 \%)$ & $19(4.6 \%)$ & 0.979 & $511(4.3 \%)$ & $62(4.2 \%)$ & 0.831 \\
\hline Diabetes mellitus & $965(29.5 \%)$ & $101(24.4 \%)$ & 0.031 & $2,427(20.4 \%)$ & $289(19.5 \%)$ & 0.400 \\
\hline LVEF $<35 \%$ & $99(3.0 \%)$ & $4(1.0 \%)$ & 0.017 & $416(3.5 \%)$ & $32(2.2 \%)$ & 0.007 \\
\hline $\begin{array}{l}\text { Preoperative haemoglobin level (mmol/l), } \\
\text { mean } \pm \text { SD }\end{array}$ & $8.0 \pm 0.8$ & $8.1 \pm 0.8$ & 0.312 & $8.8 \pm 0.8$ & $8.8 \pm 0.8$ & 0.935 \\
\hline eGFR (ml/min/1.73 m²), median (IQR) & $59.7(49.9-72.0)$ & $61.1(52.2-73.2)$ & 0.116 & $68.8(59.4-80.0)$ & $70.4(61.2-81.8)$ & 0.001 \\
\hline $\begin{array}{l}\text { Triple-vessel disease, including main stem } \\
\text { stenosis }\end{array}$ & $1,962(60.0 \%)$ & $151(36.5 \%)$ & $<0.001$ & $7,715(64.9 \%)$ & $617(41.6 \%)$ & $<0.001$ \\
\hline
\end{tabular}

Table 2 Operative data of patients undergoing on-pump coronary artery bypass $(O N C A B)$ and off-pump coronary artery bypass $(O P C A B)$ surgery

\begin{tabular}{|c|c|c|c|c|c|c|}
\hline \multirow[t]{2}{*}{ Variable } & \multicolumn{3}{|c|}{ Women $(n=3,684)$} & \multicolumn{3}{|l|}{ Men $(n=13,368)$} \\
\hline & $\begin{array}{l}\text { ONCAB } \\
(n=3,270)\end{array}$ & OРCAB $(n=414)$ & $p$-value & ONCAB $(n=11,885)$ & OPCAB $(n=1,483)$ & $p$-value \\
\hline Number of grafts, mean \pm SD & $3.5 \pm 0.9$ & $2.7 \pm 0.8$ & $<0.001$ & $3.7 \pm 0.9$ & $2.9 \pm 0.8$ & $<0.001$ \\
\hline
\end{tabular}

\section{Results}

The data of 3,684 women (3,270 ONCAB and 414 OP$\mathrm{CAB})$ and 13,368 men (11,885 ONCAB and 1,483 OP$\mathrm{CAB})$ were analysed. Preoperative patient characteristics are presented in Tab. 1. Both women and men undergoing OPCAB surgery were younger (women: ONCAB 68.8 years vs OPCAB 67.6 years, $p=0.021$; men: ONCAB 64.7 years vs OPCAB 64.0 years, $p=0.011$ ), had a relatively better LVEF (women: ONCAB $3.0 \%$ vs OPCAB $1.0 \%, p=0.017$; men ONCAB $3.5 \%$ vs OP$\mathrm{CAB} 2.2 \%, p=0.007$ ) and presented less often with triple-vessel disease (women: ONCAB $60.0 \%$ vs OPCAB 36.5\%, $p<0.001$; men: ONCAB $64.9 \%$ vs OPCAB $41.6 \%, p<0.001)$. More women undergoing OPCAB surgery were obese $(p=0.040)$, but fewer were diabetic $(p=0.031)$. More men undergoing OPCAB surgery suffered from hypertension $(p=0.016)$. The male OP$\mathrm{CAB}$ group had a higher eGFR than the male ONCAB group ( $p=0.001$ ). Tab. 2 (operative data) shows that the mean number of grafts was significantly lower in the OPCAB group compared to the ONCAB group in both women and men (both $p<0.001$ ).

Tab. 3 presents the outcomes of men and women after $\mathrm{ONCAB}$ and $\mathrm{OPCAB}$ surgery. After OPCAB surgery, both men and women received fewer red blood cell transfusions (women: ONCAB $61.6 \%$ vs OPCAB 25.4\%; men: ONCAB $22.5 \%$ vs OPCAB $10.0 \%$, both $p<0.001$ ) and had a higher postoperative haemoglobin level (women: ONCAB 6.8 vs OPCAB $7.1 \mathrm{mmol} / \mathrm{l}$, $p<0.001$; men: ONCAB 7.1 vs OPCAB $7.6 \mathrm{mmol} / \mathrm{l}$, $p<0.001$ ). In men, re-exploration for bleeding was performed significantly less frequently in the OPCAB group than in the ONCAB group $(p=0.002)$. This difference was not significant for women $(p=0.202)$. Early mortality did not differ significantly between the two types of surgery in either group (women: ONCAB $2.4 \%$ vs OPCAB $1.0 \%, p=0.057$; men: ONCAB $1.4 \%$ vs OPCAB $0.8 \%, p=0.080$ ). However, 120 -day mortality was significantly lower in women after OPCAB surgery compared to ONCAB surgery (ONCAB 3.6\% vs OPCAB $1.2 \%, p=0.010$ ), but this was not the case for men (ONCAB $2.0 \%$ vs OPCAB $1.4 \%, p=0.153$ ).

Tab. 4 presents the multivariable logistic regression analysis for mortality, red blood cell transfusion and re-exploration for bleeding, corrected for significantly different preoperative risk factors. These confounders include age, obesity, diabetes mellitus, LVEF <35\%, COPD, emergency, PVD and triple-vessel disease. In both groups, 30-day mortality was not significantly different between $\mathrm{OPCAB}$ and $\mathrm{ON}-$ $\mathrm{CAB}$ surgery [women: odds ratio $(\mathrm{OR})=0.429,95 \%$ 
Table 3 Postoperative outcomes of patients undergoing on-pump coronary artery bypass (ONCAB) and off-pump coronary artery bypass $(O P C A B)$ surgery

\begin{tabular}{|c|c|c|c|c|c|c|}
\hline \multirow[t]{2}{*}{ Variable } & \multicolumn{3}{|c|}{ Women $(n=3,684)$} & \multicolumn{3}{|l|}{ Men $(n=13,368)$} \\
\hline & $\begin{array}{l}\text { ONCAB } \\
(n=3,270)\end{array}$ & ОРСAB $(n=414)$ & $p$-value & ONCAB $(n=11,885)$ & $\mathrm{OPCAB}(n=1,483)$ & $p$-value \\
\hline CVA & $36(1.1 \%)$ & $3(0.7 \%)$ & 0.617 & $76(0.6 \%)$ & $6(0.4 \%)$ & 0.275 \\
\hline TIA/RIND & $10(0.3 \%)$ & $0(0.0 \%)$ & 0.615 & $27(0.2 \%)$ & $0(0.0 \%)$ & 0.066 \\
\hline Myocardial infarction & $98(3.0 \%)$ & $16(3.9 \%)$ & 0.337 & $260(2.2 \%)$ & $37(2.5 \%)$ & 0.449 \\
\hline Red blood cell transfusion & $2,015(61.6 \%)$ & $105(25.4 \%)$ & $<0.001$ & $2,679(22.5 \%)$ & $148(10.0 \%)$ & $<0.001$ \\
\hline Re-exploration for bleeding & $60(1.8 \%)$ & $4(1.0 \%)$ & 0.202 & $368(3.1 \%)$ & $25(1.7 \%)$ & 0.002 \\
\hline $\begin{array}{l}\text { Postoperative haemoglobin level (mmol/l), } \\
\text { mean } \pm S D\end{array}$ & $6.8 \pm 0.7$ & $7.1 \pm 0.8$ & $<0.001$ & $7.1 \pm 0.8$ & $7.6 \pm 0.9$ & $<0.001$ \\
\hline 30-day mortality & $80(2.4 \%)$ & $4(1.0 \%)$ & 0.057 & $161(1.4 \%)$ & $12(0.8 \%)$ & 0.080 \\
\hline 120-day mortality & 119 (3.6\%) & $5(1.2 \%)$ & 0.010 & $232(2.0 \%)$ & $21(1.4 \%)$ & 0.153 \\
\hline
\end{tabular}

Table 4 Multivariable logistic regression analysis of significant outcomes after off-pump coronary artery bypass (OPCAB) versus on-pump coronary artery bypass $(O N C A B)$ surgery (ONCAB surgery is the reference group)

\begin{tabular}{|c|c|c|c|c|}
\hline \multirow[t]{2}{*}{ Outcome } & \multicolumn{2}{|l|}{ Women $(n=3,684)$} & \multicolumn{2}{|l|}{ Men $(n=13,368)$} \\
\hline & $\begin{array}{l}\text { Multivariable }^{\mathrm{a}} \\
\text { OR }(95 \% \mathrm{Cl})\end{array}$ & $p$-value & $\begin{array}{l}\text { Multivariable }^{\mathrm{b}} \\
\text { OR }(95 \% \mathrm{Cl})\end{array}$ & $p$-value \\
\hline 30-day mortality & $0.429(0.155-1.189)$ & 0.104 & $0.641(0.352-1.165)$ & 0.145 \\
\hline 120-day mortality & $0.356(0.144-0.882)$ & 0.026 & $0.787(0.498-1.246)$ & 0.307 \\
\hline Red blood cell transfusion & $0.212(0.167-0.270)$ & $<0.001$ & $0.394(0.330-0.471)$ & $<0.001$ \\
\hline Re-exploration for bleeding & $0.542(0.194-1.512)$ & 0.242 & $0.524(0.347-0.791)$ & 0.002 \\
\hline
\end{tabular}

Table 5 Multivariable linear regression analysis of postoperative haemoglobin after off-pump coronary artery bypass $(O P$ $C A B)$ versus on-pump coronary artery bypass $(O N C A B)$ surgery (ONCAB surgery is reference group)

\begin{tabular}{|c|c|c|c|c|}
\hline & \multicolumn{2}{|l|}{ Women $(n=3,684)$} & \multicolumn{2}{|l|}{ Men $(n=13,368)$} \\
\hline & $\begin{array}{l}\text { Multivariable } \\
\text { B }(95 \% \text { Cl) }\end{array}$ & $p$-value & $\begin{array}{l}\text { Multivariable }{ }^{b} \\
\text { B }(95 \% \text { Cl) }\end{array}$ & $p$-value \\
\hline Postoperative haemoglobin level, mmol/l & $0.256(0.182-0.331)$ & $<0.001$ & $0.500(0.457-0.544)$ & $<0.001$ \\
\hline
\end{tabular}

confidence interval (CI) $0.155-1.189, p=0.104$; men: $\mathrm{OR}=0.641,95 \%$ CI $0.352-1.165, p=0.145]$. In women, 120-day mortality was lower in the OPCAB group compared to the ONCAB group $(\mathrm{OR}=0.356,95 \% \mathrm{CI}$ $0.144-0.882, p=0.026)$. This difference in 120-day mortality between OPCAB and ONCAB surgery was not significant in men $(\mathrm{OR}=0.787,95 \%$ CI $0.498-1.246$, $p=0.307$ ). In both men and women, OPCAB surgery was associated with fewer red blood cell transfusions $(p<0.001)$. Tab. 5 shows the multivariable linear regression analysis of postoperative haemoglobin levels, corrected for significant preoperative risk factors. After OPCAB surgery, haemoglobin levels were higher in both men and women compared to ONCAB surgery $(p<0.001)$.

\section{Discussion}

The present study investigated whether women benefit more from OPCAB than from ONCAB surgery and whether the gender difference plays a role in outcomes after these techniques. This analysis showed lower early mortality after OPCAB than after ON$\mathrm{CAB}$ surgery in both genders, although the difference was not statistically significant. After OPCAB surgery, 120-day mortality was significantly lower in women, but this difference was not significant in men. After OPCAB surgery, both women and men had higher haemoglobin levels, which was reflected in lower rates of red blood cell transfusions.

Our findings are in agreement with the findings of a meta-analysis by Attaran et al. [8]. However, in contrast to Attaran et al., who reported a higher 30-day mortality in the OPCAB group $(4.8 \%)$ than in the ON- 
CAB group $(0.7 \%)$, we found a lower 30 -day mortality in the OPCAB groups. Mack et al. [18] retrospectively analysed the data of 7,374 (3,688 pairs of) women after ONCAB or OPCAB surgery and concluded that women had higher corrected operative mortality (OR 1.733, $p=0.002$ ) after ONCAB surgery compared to OPCAB surgery. Bucerius et al. [1] showed in 2,182 consecutive patients (152 of whom underwent OP$\mathrm{CAB}$ surgery) that $\mathrm{OPCAB}$ surgery is associated with a significantly reduced 30-day mortality (OR 0.11, $p=0.032$ ) compared to ONCAB surgery.

The finding that women seem to benefit more from the elimination of cardiopulmonary bypass than men has been reported previously. Fu et al. [7], who conducted a retrospective analysis of 5,359 patients who had undergone ONCAB or OPCAB surgery, concluded that OPCAB surgery resulted in lower early mortality, especially in women. Moreover, Puskas and colleagues $[2,13,14]$ have shown repeatedly that OP$\mathrm{CAB}$ surgery is associated with significantly lower riskadjusted in-hospital mortality compared to ONCAB surgery, especially in women. The fact that no significant difference was found in 30-day mortality in women in our study might be due to the number of events being too small to reach statistical significance.

In contrast to previous studies, we evaluated 120day mortality, which was significantly lower in women undergoing OPCAB surgery than in those undergoing ONCAB surgery. Recently, it has been suggested that 30-day mortality might not be an optimal outcome measurement in cardiac surgery, as survival curves stabilise after 60-120 days, depending on the type of intervention [15]. Also, our institution is affiliated with an academic national Dutch registry programme (Meetbaar Beter) [16]. This registry was developed to improve the quality and to stimulate the transparency of data publication in cardiac care. This programme chose 120-day mortality instead of 30-day mortality as an outcome variable for quality purposes after CABG [16]. The aim of our study was limited to short-term outcomes, including both 30-day and 120-day mortality. These outcomes are directly affected by the technical aspects of the procedure, including the use of the ECC (OPCAB vs ONCAB). Longer-term outcomes such as 1-year mortality have less correlation with the operative technique, especially when using all-cause mortality as an outcome.

In the present study, we were not able to retrieve data on the cause of death in order to clarify the benefit of OPCAB in the female population. However, the difference in mortality between OPCAB and ON$\mathrm{CAB}$ surgery in women might be reflected by a difference in the number of red blood cell transfusions between the OPCAB and ONCAB groups, as red blood cell transfusion is associated with increased early mortality [19]. Early reports of OPCAB surgery [20] recommended using this technique particularly in highrisk patients who are more liable to ECC complications. Considering that women have relatively more risk factors than men, avoiding the ECC would be more beneficial in women than in men. Nuttall et al. [21] demonstrated that OPCAB surgery reduces perioperative bleeding and is associated with an overall reduction in allogenic transfusion requirement [21]. In earlier studies, female gender was an independent factor associated with perioperative blood transfusion after CABG [22, 23]. Most of these studies' populations included CABG patients with use of the ECC. In the study of Arora et al. [22], the transfusion rate was $17.3 \%$ when using the ECC and $2.7 \%$ for OPCAB cases. This means that avoiding the ECC in female patients is more beneficial with regard to transfusions than in male patients. Among others, Karkouti et al. [23] concluded that the influence of gender on the risk of transfusion is due to the smaller blood volume in women. The effect of haemodilution of the ECC is more pronounced in women, which is reflected in the fact that women benefit more from OPCAB surgery than men. Other side-effects of the ECC are possibly tolerated less well by women than by men, including coagulopathy, complement activation and neurological complications [21]. The smaller body surface area in women is an additional factor that might increase the risk of bleeding after the use of the ECC [24].

We found no significant difference regarding postoperative CVA, TIA/RIND and myocardial infarction after OPCAB and ONCAB surgery, which is in line with some previous studies performed in women [1, 18]. Although we found no statistically significant difference in postoperative CVA and TIA/RIND after OP$\mathrm{CAB}$ or ONCAB surgery, OPCAB surgery was associated with fewer neurological complications in both men and women. In our internal quality assessment, we considered such a difference to be clinically significant even in the absence of statistical significance (women: $0.7 \%$ in OPCAB compared to $1.4 \%$ in ONCAB; men: $0.4 \%$ in OPCAB compared to $0.8 \%$ in ON$\mathrm{CAB})$. This is in line with previous studies, which have shown that OPCAB surgery is associated with fewer postoperative neurological adverse events than ONCAB surgery [14, 25-27]. We found no significant difference in the incidence of postoperative myocardial infarction in our population. Previous studies reported a significantly lower incidence of myocardial infarction and major adverse cardiac events after OP$\mathrm{CAB}$ surgery compared to ONCAB surgery $[13,26]$. Our finding might be explained by the relatively small number of events.

In our population, we observed that the rate of reexploration for bleeding was significantly lower after OPCAB surgery compared to ONCAB surgery in men, but this difference was not significant in women. However, in both genders, the re-exploration rate was nearly twice as high in the ONCAB group as in the OPCAB group. In a retrospective study of 3,771 patients, Karthik and colleagues [28] reported less re-exploration for bleeding after OPCAB surgery, although the difference was not significant $3.0 \%$ in the OPCAB 
group compared to $4.4 \%$ in the OPCAB group after propensity score matching, $p=0.22$ ). In this respect, OPCAB surgery has a different heparinisation policy, apart from avoiding other adverse effects of the ECC such as complement activation [29] and coagulopathy [30].

Our study has some limitations. First, because of the retrospective design, we cannot exclude selection bias and a possible lack of variables that could have influenced the results. For instance, data on preoperative anticoagulant therapy are lacking. This factor could have affected the incidence of postoperative bleeding and consequently blood transfusion. Second, the primary outcome measures were all-cause mortality and we did not retrieve data on the cause of death. However, death within 120 days postoperatively is most likely a consequence of surgery. During the study period of almost 20 years, many surgical, ECC and anaesthetic techniques have evolved that could have influenced the outcomes as well. New implementations in ECC techniques and blood management programmes have led to better outcomes after CABG, especially in terms of postoperative blood loss and haemoglobin levels. Women have possibly benefitted more than men from such blood conservation programmes with more substantial improvement in the outcome. As we mentioned above, the choice between the two techniques was made by the individual surgeon and was not done following certain selection criteria. However, surgical bias cannot be excluded because some surgeons always performed OPCAB and the others always performed ONCAB.

In conclusion, women undergoing $\mathrm{CABG}$ seem to benefit more from OPCAB surgery than from ONCAB surgery in terms of a lower 120-day mortality. An important correlated finding is that women require fewer blood cell transfusions after OPCAB surgery.

Conflict of interest J.F. ter Woorst, A.H.T. Hoff, M.C. Haanschoten, S. Houterman, A.H.M. van Straten and M.A. Soliman-Hamad declare that they have no competing interests.

Open Access This article is distributed under the terms of the Creative Commons Attribution 4.0 International License (http://creativecommons.org/licenses/by/4.0/), which permits unrestricted use, distribution, and reproduction in any medium, provided you give appropriate credit to the original author(s) and the source, provide a link to the Creative Commons license, and indicate if changes were made.

\section{References}

1. Bucerius J, Gummert JF, Walther T, etal. Impact of off-pump coronary bypass grafting on the prevalence of adverse perioperative outcome in women undergoing coronary artery bypass grafting surgery. Ann Thorac Surg. 2005;79:807-12. discussion 812-3.

2. Puskas JD, Kilgo PD, Kutner M, Pusca SV, Lattouf O, Guyton RA. Off-pump techniques disproportionately benefit women and narrow the gender disparity in outcomes after coronary artery bypass surgery. Circulation. 2007;116:I192-I9.
3. Emmert MY, Salzberg SP, Seifert B, et al. Despite modern off-pump coronary artery bypass grafting women fare worse than men. Interact CardioVasc Thorac Surg. 2010;10:737-41.

4. Athanasiou T, Al-Ruzzeh S, Del Stanbridge R, Casula RP, Glenville BE, Amrani M. Is the female gender an independent predictor of adverse outcome after off-pump coronary arterybypass grafting? Ann Thorac Surg. 2003;75:1153-60.

5. Attia T, Koch CG, Houghtaling PL, Blackstone EH, SabikEM, Sabik JF 3rd. Does a similar procedure result in similar survival for women and men undergoing isolated coronary artery bypass grafting? J Thorac Cardiovasc Surg. 2017;153:571-579.e9.

6. Eifert S, Kilian E, Beiras-FernandezA, Juchem G, Reichart B, Lamm P. Early and mid term mortality after coronary artery bypass grafting in women depends on the surgical protocol: retrospective analysis of 3441 on- and off-pump coronary artery bypass grafting procedures. J Cardiothorac Surg. 2010;5:90.

7. Fu SP, Zheng Z, Yuan X, Zhang SJ, Gao HW, Li Y, et al. Impact of off-pump techniques on sex differences in early and late outcomes after isolated coronary artery bypass grafts. Ann Thorac Surg. 2009;87:1090-6.

8. Attaran S, Harling L, Ashrafian H, et al. Off-pump versus on-pump revascularization in females: a meta-analysis of observational studies. Perfusion. 2014;29:385-96.

9. Cartier R, Bouchot O, El-Hamamsy I. Influence of sex and age on long-term survival in systematic off-pump coronary artery bypass surgery. Eur J Cardiothorac Surg. 2008;34:826-32.

10. Nashef SA, Roques F, Michel P, Gauducheau E, Lemeshow S, Salamon R. European system for cardiac operative risk evaluation (EuroSCORE). Eur J Cardiothorac Surg. 1999;16:9-13.

11. Le Tourneau T, Pellikka PA, Brown ML, et al. Clinical outcome of asymptomatic severe aortic stenosis with medical and surgical management: importance of STS score at diagnosis. Ann Thorac Surg. 2010;90:1876-83.

12. Kowalewski M, Pawliszak W, Malvindi PG, et al. Offpump coronary artery bypass grafting improves short-term outcomes in high-risk patients compared with on-pump coronary artery bypass grafting: Meta-analysis. J Thorac Cardiovasc Surg. 2016;151:60-77.e1-58.

13. Puskas JD, Edwards FH, Pappas PA, et al. Off-pump techniques benefit men and women and narrow the disparity in mortality after coronary bypass grafting. Ann Thorac Surg. 2007;84:1447-54. discussion 1454-56.

14. Puskas JD, Kilgo PD, Lattouf OM, et al. Off-pump coronary bypass provides reduced mortality and morbidity and equivalent 10-year survival. Ann Thorac Surg. 2008;86:1139-46. discussion 1146.

15. Siregar S, Groenwold RH, de Mol BA, et al. Evaluation of cardiac surgery mortality rates: 30-day mortality or longer follow-up? Eur J Cardiothorac Surg. 2013;44:875-83.

16. Van Veghel D, Marteijn M, de Mol B, Measurably Better Study Group (The Netherlands) and Advisory Board. First results of a national initiative to enable quality improvement of cardiovascular care by transparently reporting on patient-relevant outcomes. Eur J Cardiothorac Surg. 2016;49:1660-9.

17. Levey AS, Coresh J, Greene T, et al. Chronic kidney disease epidemiology collaboration. Using standardized serum creatinine values in the modification of diet in renal disease study equation for estimating glomerular filtration rate. Ann Intern Med. 2006;145:247-54.

18. Mack MJ, Brown P, Houser F, et al. On-pump versus off-pump coronary artery bypass surgery in a matched 
sample of women: a comparison of outcomes. Circulation. 2004;110:II-1-II-6.

19. Murphy GJ, Reeves BC, Rogers CA, Rizvi SI, Culliford L, Angelini GD. Increased mortality, postoperative morbidity, and cost after red blood cell transfusion in patients having cardiac surgery. Circulation. 2007;116:2544-52.

20. Buffolo E, de Andrade SJC, Rodrigues Branco JN, Teles CA, Aguiar LF, Gomes WJ. Coronary artery bypass grafting without cardiopulmonary bypass. Ann Thorac Surg. 1996;61:63-6.

21. Nuttall GA, Erchul DT, Haight TJ, et al. A comparison of bleeding and transfusion in patients who undergo coronary artery bypass grafting via sternotomy with and without cardiopulmonary bypass. J Cardiothorac Vasc Anesth. 2003; $17: 447-51$.

22. Arora RC, Légaré JF, Buth KJ, Sullivan JA, Hirsch GM. Identifying patients at risk of intraoperative and postoperative transfusion in isolated CABG: Toward selective conservation strategies. Ann Thorac Surg. 2004;78:1547-55.

23. Karkouti K, Cohen MM, McCluskey SA, Sher GD. A multivariablemodel for predicting the need for blood transfusion in patients undergoing first-time elective coronary bypass graft surgery. Transfusion. 2001;41:1193-203.

24. van Straten AH, Kats S, Bekker MW, et al. Risk factors for red blood cell transfusion after coronary artery bypass graft surgery. J Cardiothorac Vasc Anesth. 2010;24:413-7.
25. Hannan EL, Wu C, Smith CR, et al. Off-pump versus on-pump coronary artery bypass graft surgery: differences in short-term outcomes and in long-term mortality and need for subsequent revascularization. Circulation. 2007;116:1145-52.

26. Reston JT, Tregear SJ, Turkelson CM. Meta-analysis of shortterm and mid-term outcomes following off-pump coronary artery bypass grafting. Ann Thorac Surg. 2003;76:1510-5.

27. Moss E, Puskas JD, Thourani VH, et al. Avoiding aortic clamping during coronary artery bypass grafting reduces postoperative stroke. J Thorac Cardiovasc Surg. 2015;149:175-80.

28. Karthik S, Musleh G, Grayson AD, et al. Effect of avoiding cardiopulmonary bypass in non-elective coronary artery bypass surgery: a propensity score analysis. Eur J Cardiothorac Surg. 2003;24:66-71.

29. Bruins P, te Velthuis H, Yazdanbakhsh AP, et al. Activation of the complement system during and after cardiopulmonary bypass surgery: postsurgery activation involves C-reactive protein and is associated with postoperative arrhythmia. Circulation. 1997;96:3542-8.

30. Paparella D, Brister SJ, Buchanan MR. Coagulation disorders of cardiopulmonary bypass: a review. Intensive Care Med. 2004;30:1873-81. 